Hugo Alberto Nakamoto

\title{
Avaliação da sensibilidade cutânea em pacientes com síndrome do túnel do carpo relacionada à hemodiálise
}

Dissertação apresentada à Faculdade de Medicina da Universidade de São Paulo para obtenção do título de Mestre em Ciências

Área de concentração: Cirurgia Plástica

Orientador: Prof. Dr. Marcus Castro Ferreira

São Paulo

2009 
A meus pais, Shugoro e Iracema, pela dedicação irrestrita aos filhos e ensinamentos de vida. A minhas tias queridas, exemplos de vida e caráter. A minha esposa Flavia, pelo apoio incondicional e amor durante todos esses anos. 


\section{Agradecimentos}

Ao Prof. Dr. Marcus Castro Ferreira, professor na mais clara acepção da palavra. Homem de visão privilegiada, a quem dedico sincero respeito e admiração.

Ao Dr. Paulo Tuma Junior, meu grande mestre dentro da Cirurgia da Mão. Agradeço a acolhida dentro do Grupo de Mão, e também a convivência agradável e estimulante durante todos esses anos.

Ao Prof. Dr. Marcelo Sacramento Cunha, pelos grandes ensinamentos de metodologia científica e pela amizade e confiança sempre demonstrados.

Ao Dr. Marcelo Rosa Rezende, pelos grandes ensinamentos em Cirurgia da Mão.

Aos Drs. Dimas André Milcheski, Nélio Watanabe Aguilera e José Carlos Faes da Silva, meus grandes amigos e colegas no Grupo de Mão.

Ao Dr. Walfredo Cherubini Fogaça, pela grande ajuda no início de minha carreira como cirurgião plástico.

Ao Dr. Jorge Ishida, pelos preciosos ensinamentos dentro de nossa especialidade, durante e depois da residência médica. 
Aos Drs. Alexandre Mendonça Munhoz, Dov Charles Goldenberg, Fábio de Freitas Busnardo, Luis Henrique Ishida e Luiz Carlos Ishida, exemplos mais próximos dentro da profissão e pessoas nas quais sempre me espelhei.

Aos Drs. Alberto Yoshikazu Okada, Eduardo Montag e Fabio Lopes Saito, grandes amigos para todas as horas.

Aos meus irmãos, os Drs. João Carlos Nakamoto e Marcio Luis Nakamoto, com quem posso contar sempre que preciso.

Ao acadêmico Francisco Tustumi, pela grande ajuda na fase final desta tese.

A todos os colegas e funcionários da Disciplina de Cirurgia Plástica, que dia a dia constroem um ambiente de excelência e amizade; do qual tenho muito orgulho de fazer parte. 


\section{Sumário}

Lista de abreviaturas e siglas

Lista de símbolos

Lista de figuras

Lista de tabelas

Resumo

Summary

1. INTRODUÇÃO

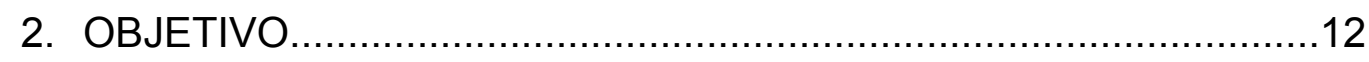

3. MÉTODOS

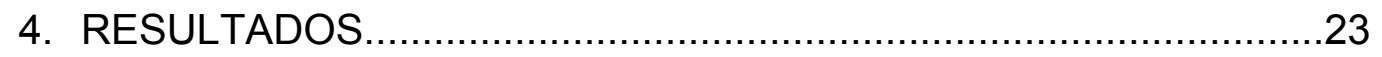

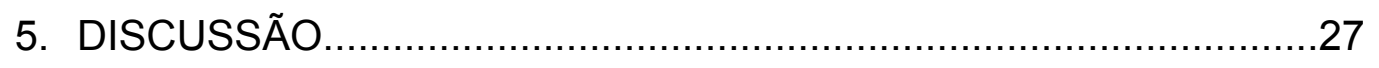

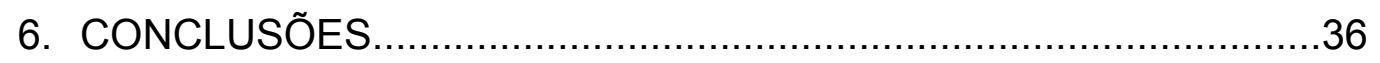

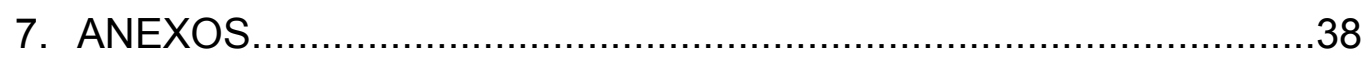

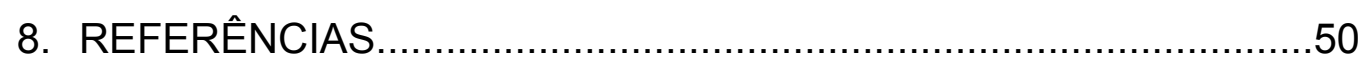




\section{Lista de abreviaturas e siglas}

$\begin{array}{ll}\text { CAPPesq } & \text { Comissão de Ética para análise de projetos de } \\ & \text { pesquisa do Hospital das Clínicas da Faculdade de } \\ \text { Co. } & \text { Medicina da Universidade de São Paulo } \\ \text { Dr. } & \text { "Company" } \\ \text { ed. } & \text { doutor } \\ \text { et al. } & \text { edição } \\ \text { p. } & \text { página } \\ \text { Prof. } & \text { professor } \\ \text { PSSD- } & \text { "Pressure Specified- Sensory Device } \\ 1 \text { PE } & \text { teste estático de um ponto } \\ 1 \text { PD } & \text { teste dinâmico de um ponto dinâmico } \\ 2 \text { PE } & \text { teste estático de dois pontos } \\ 2 \text { PD } & \text { teste dinâmico de dois pontos }\end{array}$




\section{Lista de símbolos}

$\begin{array}{ll}\mathrm{g} / \mathrm{mm}^{2} & \text { grama por milímetro quadrado } \\ \mathrm{Hz} & \text { hertz } \\ \mathrm{mm} & \text { milímetro } \\ \mathrm{mmHg} & \text { milímetro de mercúrio } \\ \mu \mathrm{m} & \text { micrometro } \\ \mathrm{p} & \text { nível de significância } \\ < & \text { menor que } \\ > & \text { maior que } \\ \mathrm{TM} & \text { "Trademark" } \\ \% & \text { porcentagem } \\ \mathrm{X} & \text { versus } \\ \alpha & \text { alfa } \\ \beta & \text { beta } \\ \gamma & \text { gama }\end{array}$




\section{Lista de figuras}

Figura 1 Transdutor de pressão do PSSD...............................18

Figura 2 Visão geral do PSSD............................................18

Figura 3 Posicionamento do examinador e do paciente

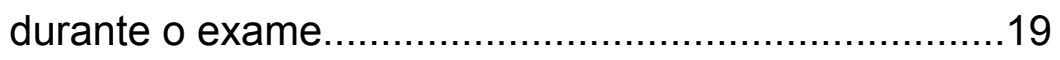

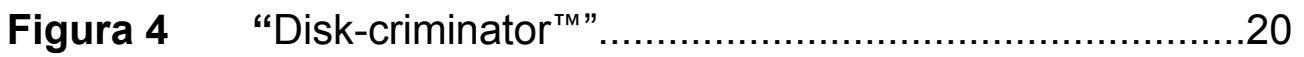




\section{Lista de tabelas}

Tabela 1 Valores dos limiares cutâneos de pressão

para pacientes do Grupo 1.

Tabela 2 Valores dos limiares cutâneos de pressão

para pacientes dos Grupos 2 e 3...............................25

Tabela 3 Sensibilidade para o diagnóstico de síndrome do túnel do carpo utilizando o PSSD para os grupos 2 e $3 \ldots \ldots \ldots \ldots . . .26$

Tabela 4 Valores de $\mathrm{p}$ para o teste exato de Fisher, utilizado para comparação entre os grupos 2 e $3 \ldots \ldots \ldots \ldots \ldots \ldots \ldots \ldots \ldots \ldots \ldots . . .26$ 


\section{Resumo}

\section{NAKAMOTO, H.A. Avaliação da sensibilidade cutânea em pacientes com}

síndrome do túnel do carpo relacionada à hemodiálise. São Paulo, 2009. Dissertação(Mestrado)- Faculdade de Medicina, Universidade de São Paulo.

A síndrome do túnel do carpo é a neuropatia compressiva mais comum do membro superior. Cerca de 5\% dos pacientes com insuficiência renal crônica submetidos à hemodiálise apresentam esta condição. A história clínica e o exame físico continuam sendo as ferramentas mais adequadas para seu diagnóstico. Testes complementares têm sido usados para auxiliar o diagnóstico, dentre eles os de avaliação da sensibilidade cutânea. O objetivo deste estudo é descrever a utilização do PSSD ("Pressure- specified sensory device") como auxiliar ao diagnóstico de síndrome do túnel do carpo. O PSSD consiste de aparelho que incorpora transdutor de pressão com duas extremidades rombas e com regulagem de distância entre elas, acoplado a um computador capaz de determinar os limiares cutâneos de pressão. Para tanto, os pacientes foram divididos em três grupos: Grupo 1- grupo controle, pacientes sem neuropatia no membro superior ou insuficiência renal. Grupo 2- pacientes com síndrome do túnel do carpo e insuficiência renal crônica submetidos à hemodiálise. Grupo 3- pacientes com síndrome do túnel do carpo sem 
insuficiência renal. Os resultados demonstraram haver um maior benefício do uso do PSSD para o diagnóstico da síndrome para os pacientes do grupo 2 em dois dos quatro parâmetros avaliados quando comparados com os pacientes do grupo 3. 


\title{
Summary
}

\begin{abstract}
NAKAMOTO, H.A. Cutaneous sensibility testing in patients with hemodialysis- associated carpal tunnel syndrome. São Paulo, 2009. Dissertação(Mestrado)- Faculdade de Medicina, Universidade de São Paulo.
\end{abstract}

Carpal Tunnel Syndrome is the most common compressive neuropathy of the superior limb. Close to $5 \%$ of the patients with chronic renal insufficiency who need hemodialysis have carpal tunnel syndrome. There are controversies about the most reliable test to complement the clinical history and physical exam that remain as the most adequate tools for the diagnosis. Evaluation of sensory thresholds is being used as one of these tests. The aim of this study is to use the PSSD (Pressure-specified sensory device) for the diagnosis of carpal tunnel syndrome. The PSSD is a tool that incorporates a pressure transducer with two prongs, linked to a computer capable of measuring the cutaneous pressure thresholds referred by the patient. The patients were divided in three groups: Group 1- patients with neither superior limb neuropathy nor renal insufficiency. Group 2- patients with carpal tunnel syndrome related to chronic renal insufficiency under hemodialysis. Group 3- patients with carpal tunnel syndrome but no renal insufficiency. The results showed, for two of the four parameters 
measured, that group 2 had more benefits for the diagnosis of carpal tunnel syndrome than group 3 . 


\section{Introdução}




\section{Introdução}

A síndrome do túnel do carpo é a neuropatia compressiva mais comum do membro superior, sendo caracterizada clinicamente por parestesias, formigamentos e dor na distribuição do nervo mediano na mão. ${ }^{1}$ Foi descrita inicialmente por Sir James Paget; em paciente com neurite pós traumática após "formação excessiva de calo ósseo decorrente de fratura de Colles". 2

Em 1913, Marie e Foix * apud Dellon; demonstraram através de microscopia atenuação na bainha de mielina das fibras do nervo mediano na altura do punho em autópsia realizada em paciente de 80 anos com atrofia tenar bilateral. Os autores sugeriram que a liberação cirúrgica do ligamento carpal transverso poderia ter melhorado a sintomatologia. ${ }^{3}$

No entanto, foram Phalen et al. que primeiro descreveram com detalhes a síndrome, notando que "o nervo mediano é facilmente comprimido por qualquer condição que aumente o volume das estruturas dentro do canal do carpo". 4

Zachary em 1945 tentou pela primeira vez tratamento cirúrgico para o descompressão do túnel do carpo. ${ }^{5}$

* Marie P; Foix, C. Atrophie isolee de l'eminence thenar d'origine neiritique. Role du ligament annulaire anteireur du cape dans la pathogenie de la lesion. Rev Neurol 1913; 26: 647-649 
O túnel do carpo é uma estrutura fibro-óssea que conecta superficialmente o antebraço distal ao compartimento médio profundo da palma da mão. O seu assoalho é composto pelos ossos do carpo. O gancho do hamato, o piramidal e o pisiforme constituem sua borda ulnar, enquanto seu aspecto radial é constituído pelo trapézio, escafóide e a fáscia do flexor radial do carpo. ${ }^{6} \mathrm{O}$ teto do canal do carpo é formado pela fáscia profunda do antebraço e pelo ligamento carpal transverso, assim como pela aponeurose proximal dos músculos tenares e hipotenares.

O conteúdo do túnel do carpo consiste no nervo mediano, posicionado superficial e anteriormente, e em nove tendões flexores: o flexor longo do polegar, quatro flexores profundos e quatro flexores superficiais dos dedos longos. Variações anatômicas deste conteúdo foram descritas: existência de uma artéria mediana, músculos lumbricais ou ventres musculares dos flexores superficiais e profundos estendendo-se para dentro do canal. ${ }^{7,8,9}$

Suas paredes ósseas não são inteiramente rígidas uma vez que os ossos do carpo se movem em relação aos outros com a movimentação da mão. Flexionando-se o punho há uma diminuição marcante do diâmetro proximal do túnel através da aproximação do retináculo dos flexores radiais. Em extensão plena, o semilunar é pressionado para o interior do túnel, também diminuindo suas dimensões. Estudos de Baumann et al. demonstraram que a pressão média dentro do túnel do carpo na posição neutra do punho é de $2 \mathrm{mmHg}$. Em flexão máxima, a pressão aumenta para $42 \mathrm{mmHg}$. A pressão em extensão plena é de $3 \mathrm{mmHg} .{ }^{10}$ 
Qualquer aumento da pressão de caráter constante dentro do canal do carpo pode comprometer a função do nervo mediano. Isto se deve não apenas à pressão mecânica exercida mas também devido à interrupção do aporte sanguíneo para o mesmo. Rydevik e Lundborg demonstraram que a aplicação de $20 \mathrm{mmHg}$ de pressão ao nervo periférico é suficiente para diminuir o fluxo sanguíneo para o interior do mesmo, com edema intraneural e início do processo de compressão nervosa crônica. ${ }^{11}$

A compressão crônica do nervo implica que haja uma região sujeita à pressão por período de tempo suficiente para causar alterações fisiopatológicas. Estas alterações serão dependentes da intensidade da compressão e também do tempo em que esta é exercida. Modelos experimentais demonstraram que a compressão exercida cronicamente causa alterações na barreira hematonervosa, permitindo a ocorrência de edema endoneurial. Se a compressão é mantida por um período de 6 meses, inicia-se processo de fibrose epineural e perineural, assim como adelgaçamento da bainha de mielina. Isto é acompanhado pela diminuição da velocidade de condução nervosa. Após cerca de 1 ano, pode se iniciar processo de degeneração walleriana. ${ }^{12,13}$

Warren e Otieno foram os primeiros a descrever a relação existente entre a hemodiálise e a síndrome do túnel do carpo. No entanto, em seu estudo inicial os mecanismos implicados na gênese desta condição não eram muito bem conhecidos. Os autores propuseram que a pressão venosa aumentada na mão, decorrente da fístula arteriovenosa, iniciaria o processo. ${ }^{14,15}$ 
A identificação da proteína $\beta 2$ - microglobulina em tecidos perineurais, sinoviais e em paratendões estabeleceu a relação entre a síndrome do túnel do carpo e a amiloidose associada a hemodiálise.$^{16}$ Sabe-se que cerca de $5 \%$ dos pacientes com insuficiência renal crônica submetidos à hemodiálise apresentam síndrome do túnel do carpo. ${ }^{17}$

A história clínica e o exame físico continuam sendo os critérios mais adequados para o diagnóstico de síndrome do túnel do carpo. Em relação aos testes complementares, há controvérsias a respeito do exame mais confiável, mas a maioria dos especialistas concorda não existir padrão-ouro. No entanto o diagnóstico mais completo é considerado essencial para o estabelecimento do tratamento, seja ele conservador ou cirúrgico; e para validação de dados epidemiológicos. $^{1,18,19,20}$

Os testes eletrodiagnósticos são considerados o modo mais comum de encontrar objetivamente evidência da síndrome.$^{21}$ No entanto, cerca de 10 a $15 \%$ dos pacientes com estudos de condução nervosa normais apresentam evidência clínica da síndrome, assim como alívio dos sintomas com a liberação cirúrgica do túnel do carpo. ${ }^{1,18}$

Segundo Hentz e Lalonde, "existem fortes evidências de que realizar estudos eletrodiagnósticos em todos os pacientes é desnecessário e há significativo número de falsos positivos e falso negativos". ${ }^{18}$

Jablecki et al. em revisão da literatura publicada pela Associação Americana de Medicina Eletrodiagnóstica concluiram que estudos de condução 
nervosa do nervo mediano confirmam o diagnóstico de síndrome do túnel do carpo em cerca de $85 \%$ dos casos. ${ }^{22}$

Szabo et al. em um estudo prospectivo com 100 pacientes consecutivos concluíram que a história clínica e o exame físico como método primário para o diagnóstico de síndrome do túnel do carpo são suficientes na maioria das vezes. Em seu estudo, os critérios diagnósticos utilizados com maior sensibilidade foram o teste de compressão de Durkan e os monofilamentos de Semmes-Weistein ; enquanto que os testes mais específicos foram os diagramas de mão e o sinal de Tinel. ${ }^{20}$

Graham, em estudo prospectivo e duplo cego, demonstrou que o valor adicionado pelos estudos de condução nervosa para o diagnóstico de síndrome do túnel do carpo era muito pequeno para a vasta maioria de pacientes encaminhados a um centro de atenção terciária. ${ }^{23}$

A sensibilidade normal da mão é de extrema importância para a sua função, e vários testes têm sido utilizados para sua avaliação. Estes exames foram introduzidos na prática clínica por Weber em 1835(Discriminação de dois pontos) e por von Frey* em 1905, ambos para avaliação de lesões traumáticas de nervos nos membros superiores. ${ }^{1,18}$

*Weber, E. Ueber den Tatsinn. Archive fur Anatomy und Physiology. Wissenshaft Medical Muller's Archives. 1835; 1: 152.

Von Frey, M. The distribution of afferent nerves in the skin. $J$ am med assoc. 1906, 47: 152159. 
No entanto, as bases fisiológicas para o entendimento da sensibilidade cutânea foram estabelecidas apenas anos depois.

Segundo Gasser e Erlanger, ${ }^{24}$ as fibras nervosas podem ser classificadas em:

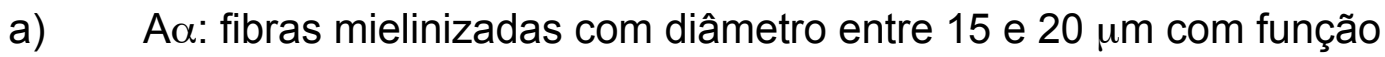
motora.

b) $A \beta$ : fibras mielinizadas com diâmetro entre 10 e $15 \mu \mathrm{m}$ com função de sensibilidade(percepção ao toque).

c) A $\gamma$ : fibras mielinizadas com diâmetro entre 2 e $5 \mu \mathrm{m}$ com função de sensibilidade( dor e temperatura).

d) C: fibras não mielinizadas com diâmetro entre 0,5 e 1,5 $\mu \mathrm{m}$ com função de sensibilidade (dor em queimadura).

O grupo de fibras $A \beta$ apresenta propriedades fisiológicas de adaptação úteis na percepção. Mountcastle et al. ${ }^{25}$ classificam este grupo de fibras em dois subgrupos:

a) Fibras de adaptação lenta: são aquelas que começam a gerar impulsos a partir de um estímulo físico e continuam a gerá-los enquanto este estímulo persistir. Este estímulo é interpretado como tato constante e estático. Caso a intensidade da força aplicada aumente, há um aumento da frequência de impulsos e isto será percebido como aumento da pressão exercida. Relacionam-se com as células de Merkel, que são os receptores cutâneos responsáveis pela percepção do estímulo do tipo pressão na pele glabra. 
b) Fibras de adaptação rápida: são aquelas que geram somente um ou dois impulsos ao serem estimuladas e param de gerá-las em seguida. Estas fibras somente originarão novos impulsos quando o estímulo for descontinuado e realizado novamente. Quando ocorre aumento da intensidade do estímulo, não há aumento da geração de impulsos e portanto este não é percebido. Estas fibras são responsáveis pela percepção de movimento e vibração. Os corpúsculos de Paccini e Meissner são os receptores associados a estas fibras na pele glabra.

Os testes de sensibilidade cutânea podem auxiliar no diagnóstico da síndrome do túnel do carpo em situações onde o exame físico causa dúvida, mas principalmente quando existem resultados contraditórios entre o exame físico e a eletroneuromiografia. No entanto, há problemas como a falta de universalização dos testes utilizados, a falta de precisão e normatização de alguns tipos de teste e a ausência de consenso quanto às informações relevantes dentro das modalidades de sensibilidade a serem avaliadas. ${ }^{26}$

Segundo Dellon et al., a história dos testes de avaliação da sensibilidade é feita por uma progressiva melhora na instrumentação utilizada, evoluindo dos testes qualitativos para quantitativos em uma mesma modalidade. ${ }^{27}$

Há dois tipos de exame quantitativo da sensibilidade cutânea: testes de limiar cutâneo de pressão e o teste de discriminação de dois pontos.

O limiar cutâneo de pressão determina a função tátil da mão e leva em consideração a relação da fibra nervosa com seu receptor cutâneo. O limiar é 
uma percepção medida em resposta a um estímulo definido, representando um ponto ao longo de um espectro físico abaixo do qual não há detecção da existência do evento e acima do qual existe consciência de sua existência. A discriminação de dois pontos por sua vez, relaciona-se com a densidade de fibras. Ambos os aspectos são importantes e não podem ser dissociados. ${ }^{27}$ Weber apud Dellon* descreveu o teste de discriminação de dois pontos consistindo essencialmente de uma avaliação para determinar a distância mínima na qual o paciente pode discriminar estar sendo tocado por dois pontos. $^{27}$

Moberg difundiu a importância da discriminação de dois pontos como medida relacionada à função da mão, ${ }^{28}$ o que foi confirmado posteriormente por diversos autores. ${ }^{29,30}$ Há uma correlação entre a discriminação de dois pontos e a densidade de fibras de uma determinada região, isto é, quanto maior a densidade de fibras menor é a distância reconhecida como dois pontos separados. $^{27,29,30}$

No entanto, a impossibilidade de controlar a pressão exercida durante o exame leva a dificuldades de comparação dos dados de diferentes instituições, pacientes e às vezes até diferentes avaliações de um mesmo paciente..$^{31,32}$

*Weber, E. Ueber den Tatsinn. Archive fur Anatomy und Physiology. Wissenshaft Medical Muller's Archives. 1835, 1: 152-159. 
Além disso ela não pode ser o único exame a ser realizado, já que a perda de fibras nervosas normalmente é evento tardio em doenças como as neuropatias compressivas. ${ }^{27,} 32$

O teste de limiar cutâneo de pressão mais conhecido é o de SemmesWeinstein. Este exame permite uma estimativa de intervalos de percepção do limiar cutâneo de pressão dos pacientes. Esta limitação do teste( a incapacidade de obtenção de resultados contínuos) é um problema inerente ao exame, que pode por exemplo, deixar de detectar uma alteração da sensibilidade cutânea caso esta permaneça dentro do mesmo intervalo obtido anteriormente. $^{27,33}$

Levin et al. avaliaram a calibração e as propriedades mecânicas dos monofilamentos de Semmes-Weinstein, além de determinar os cálculos de estresse de deformação e força do material. Os autores concluíram que erros de aplicação e interpretação podem levar a grande variabilidade e consequentemente influenciar os resultados. ${ }^{34}$

Pagel et al. em estudo duplo-cego com 119 pacientes portadores de sintomas compatíveis com síndrome do túnel do carpo compararam o diagnóstico realizado pelo teste de monofilamentos com o diagnóstico realizado por testes eletrodiagnósticos. O estudo concluiu não haver utilidade dos monofilamentos para o diagnóstico da síndrome do túnel do carpo. ${ }^{35}$

Em 1992, Dellon et al. introduziram na prática clínica teste computadorizado de avaliação da sensibilidade cutânea, o PSSD( " Pressure- 
Specified Sensory Device ${ }^{T M}$ ”), que mede limiares cutâneos de pressão. Este aparelho possibilita a aferição de parâmetros de percepção de pressão não disponíveis previamente. O PSSD incorpora transdutor de pressão com duas extremidades rombas e com regulagem de distância entre elas, acoplado a um computador capaz de determinar os limiares cutâneos de pressão. Isto permite a medida contínua da pressão entre 0,1 e $100 \mathrm{~g} / \mathrm{mm}^{2}$ e simultaneamente a capacidade de discriminação de dois pontos, com sensibilidade e precisão maiores do que outros testes desenvolvidos previamente. ${ }^{27,36}$

O PSSD foi utilizado para diagnóstico de síndromes compressivas do membro superior (síndrome do túnel do carpo e síndrome do túnel cubital) em 1997, em estudo com 72 pacientes com história clínica sugestiva, exame físico (testes provocativos e sinal de Tinel positivos) e percepção anormal do diapasão de $256 \mathrm{~Hz}$. Este estudo demonstrou que o primeiro parâmetro alterado nas neuropatias compressivas é o teste estático de dois pontos, sugerindo que para a detecção precoce das síndromes compressivas, este é mais adequado que o teste de limiar cutâneo de pressão de um ponto estático(correspondente ao teste dos monofilamentos de SemmesWeinstein). ${ }^{27,37}$

Em nosso meio, o PSSD foi introduzido no Hospital das Clínicas da Faculdade de Medicina da Universidade de São Paulo em 1998 e tem sido usado para avaliação de sensibilidade cutânea em diferentes condições de pós- 
operatório de cirurgia plástica e em neuropatias de membros inferiores de pacientes diabéticos. ${ }^{38,39,40,41}$ 
Objetivo 


\section{Objetivo}

Determinar, com o PSSD, os limiares cutâneos de pressão no território do nervo mediano. Pacientes com insuficiência renal submetidos cronicamente a hemodiálise e portadores de síndrome do túnel do carpo foram comparados com pacientes que apresentaram a síndrome mas não tinham insuficiência renal. 


\section{Métodos}




\section{Métodos}

Foram realizadas avaliações da sensibilidade cutânea em pacientes acompanhados no Grupo de Cirurgia da Mão da Disciplina de Cirurgia Plástica do Hospital das Clínicas da Faculdade de Medicina da Universidade de São Paulo. Este projeto foi aprovado pela Comissão de Ética para Análise de Projetos de Pesquisa do Hospital das Clínicas da Faculdade de Medicina da Universidade de São Paulo, sob o número 1247, projeto CAPPesq 1088/07.

Os pacientes com suspeita diagnóstica de síndrome do túnel do carpo foram submetidos a um questionário padrão, contendo dados de história e de exame físico, e aplicados sempre pelo mesmo profissional. Para inclusão no protocolo foram coletados dados da história clínica( parestesia noturna) e achados compatíveis no exame físico (sinal de Tinel, sinal de Phalen). Pacientes com história clínica inconsistente e exame físico duvidoso foram encaminhados para a realização de testes eletrodiagnósticos.

O padrão-ouro para diagnóstico da síndrome do túnel do carpo foi considerada a melhora dos sintomas no pós operatório.

Os pacientes foram divididos em três grupos:

Grupo 1- Pacientes sem patologia comprovada, tanto renal quanto em nervos periféricos dos membros superiores, considerados grupo controle.

Grupo 2- Pacientes com síndrome do túnel do carpo associada à insuficiência renal crônica, e em programa de hemodiálise. 
Grupo 3- pacientes com síndrome do túnel do carpo mas sem diagnóstico de insuficiência renal.

Foram excluídos deste protocolo pacientes com síndrome do túnel do carpo já operados previamente, pacientes com história prévia de lesão do neurônio motor superior , pacientes com incapacidade de realizar as funções motoras necessárias para a realização do teste e pacientes com incapacidade de compreender as instruções para o teste.

Cinquenta e três pacientes foram incluídos no estudo. Destes, 29 apresentavam síndrome do túnel do carpo. No grupo 1(controle) foram incluídos 24 pacientes, com 45 mãos avaliadas. No grupo 2 foram estudados 14 pacientes, com 22 mãos avaliadas. No grupo 3, foram incluídos 15 pacientes com 21 mãos examinadas.

No grupo controle, a idade média foi de 41,4 anos, variando de 19 a 72 anos. Nos grupos 2 e 3, a idade média dos pacientes foi de 51,1 anos, variando de 28 a 77 anos (51,7 anos de idade média para o grupo 2 e 50,5 anos para o grupo 3).

Os pacientes do grupo 2 apresentaram tempo médio de hemodiálise de 16,2 anos, variando de 8 a 28 anos. Todos os pacientes apresentavam síndrome do túnel do carpo no lado com a fístula, sendo que $57 \%$ deles apresentavam-na bilateralmente. Nos pacientes do grupo 3, 46\% apresentavam a síndrome bilateralmente.

O sinal de Tinel positivo, definido como aparecimento de parestesias na distribuição do nervo mediano produzidas pelo percussão deste nervo na região 
do punho, esteve presente em $83 \%$ dos pacientes $(90,9 \%$ dos pacientes do grupo 2 e em $75 \%$ dos pacientes do grupo 3 ).

O sinal de Phalen positivo, definido como aparecimento ou aumento de parestesias no território do nervo mediano em 60 segundos após flexão dos punhos esteve presente em $80,9 \%$ do total dos pacientes. Nos pacientes submetidos à hemodiálise, esteve presente em $95,4 \%$, enquanto que nos indivíduos sem doença renal, esteve presente em apenas $65 \%$ das vezes.

A parestesia noturna esteve presente em $74 \%$ das ocasiões; e foi referida por $78 \%$ dos pacientes do grupo 2 e por $69,2 \%$ dos pacientes do grupo 3.

A atrofia tenar foi verificada em apenas $11,1 \%$ dos pacientes.

Os testes de sensibilidade foram executados através da utilização do PSSD( "Pressure-Specified Sensory Device ${ }^{\text {TM }}$ - NK Biotechnical Engineering Co."). O PSSD é um instrumento aparelhado com duas extremidades de metal em paralelo de pontas rombas( Figura 1), sendo que a distância entre estas extremidades pode ser ajustada pelo examinador( de 2,5 a $20 \mathrm{~mm}$ ). Estas extremidades por sua vez estão ligadas a transdutores de pressão, que permitem a verificação da força de aplicação sobre a pele de cada um deles ( para medidas de pressão de um ponto), ou de ambos (para medidas de dois pontos). O PSSD está ligado a um computador, permitindo a visualização dos dados na tela e também sua análise e armazenamento(Figura 2). 


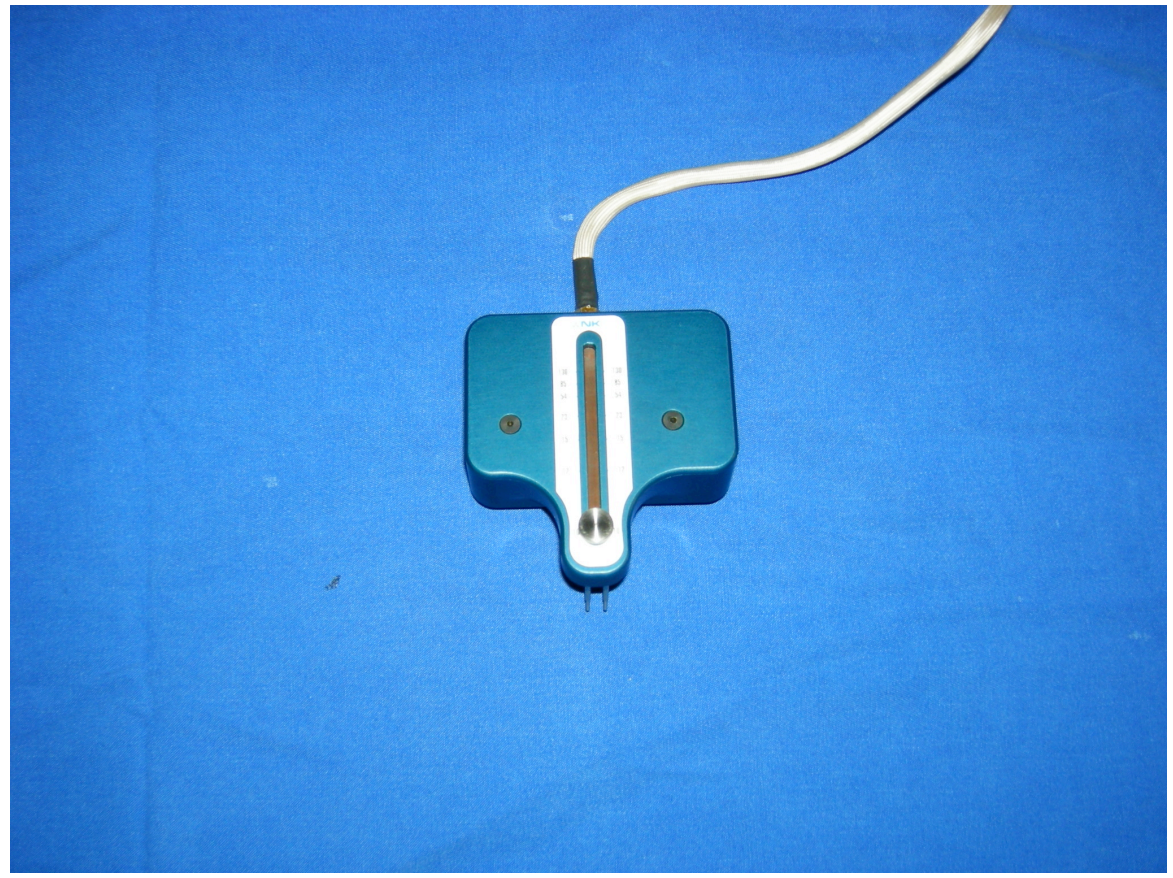

Figura 1- Transdutor de pressão do PSSD

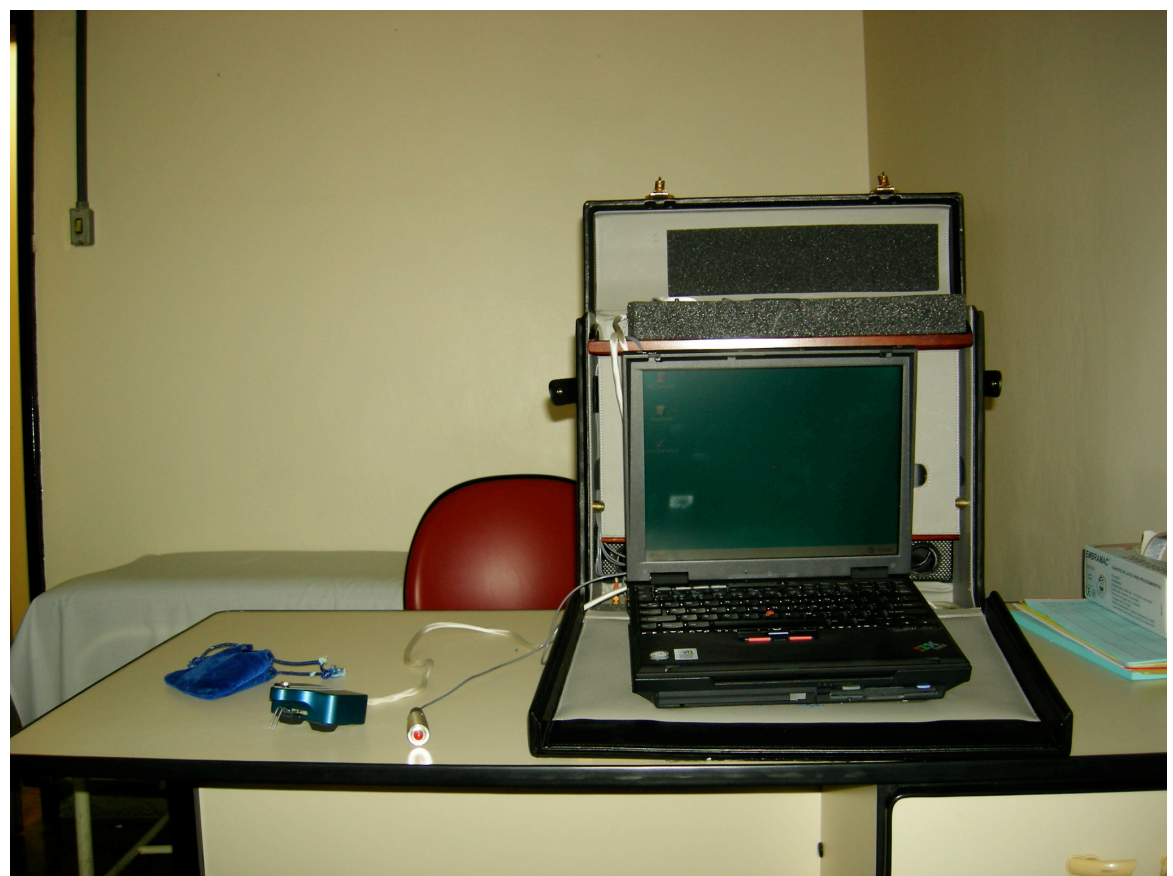

Figura 2- Visão geral do PSSD: Da esquerda para a direita vemos o transdutor de pressão, o aviso sonoro e o computador no qual os dados são analisados e armazenados. 
O teste é realizado em ambiente calmo, onde o paciente esteja livre de distrações. Este senta-se em frente ao examinador, com a mão a ser examinada descansando confortavelmente sobre a mesa. O paciente não deve ver a tela do computador. O examinador explica como o exame é realizado, que não irá machucar ou causar dor e inicia os testes. A região escolhida e padronizada para o teste de sensibilidade no território do nervo mediano foi a polpa digital do dedo indicador(Figura 3).

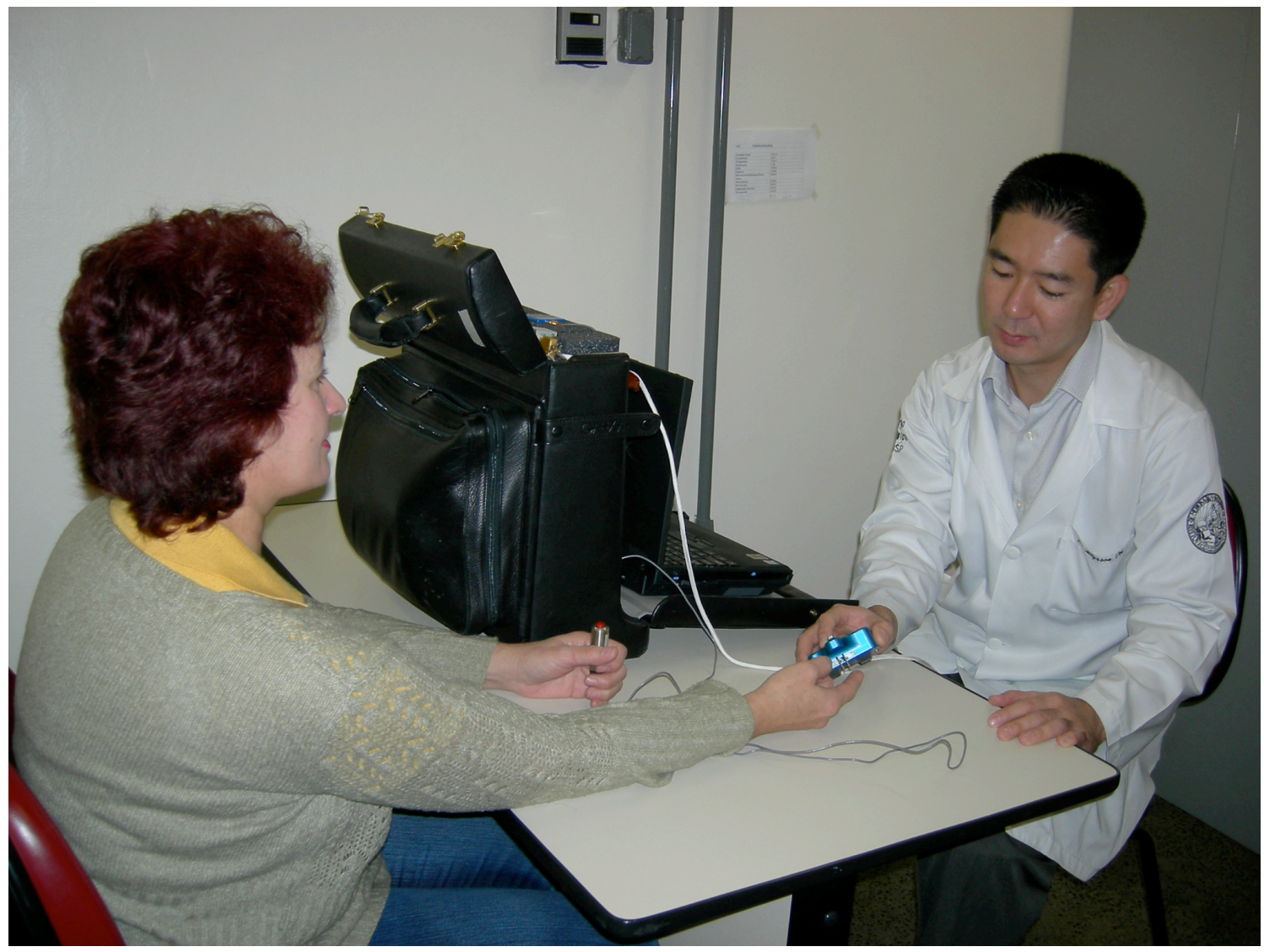

Figura 3- Posicionamento do examinador e do paciente durante o exame. $\mathrm{O}$ paciente não visualiza a tela do computador 
O exame iniciou-se com a determinação da discriminação de dois pontos, utilizando o "Disk-criminator ${ }^{\mathrm{TM}}$ " ( Figura 4). Foi considerada a distância correta quando o paciente acertou sete em cada dez respostas( por exemplo, foi considerada de $3 \mathrm{~mm}$ a discriminação de dois pontos caso o paciente tenha sido capaz de reconhecer que existem duas pontas tocando sua polpa digital em sete tentativas de dez). ${ }^{42} \mathrm{O}$ examinador deve tomar cuidado para não tocar uma extremidade do dispositivo por vez ou para não colocar muito mais pressão em uma das extremidades, o que facilita para o paciente o reconhecimento de duas pontas.

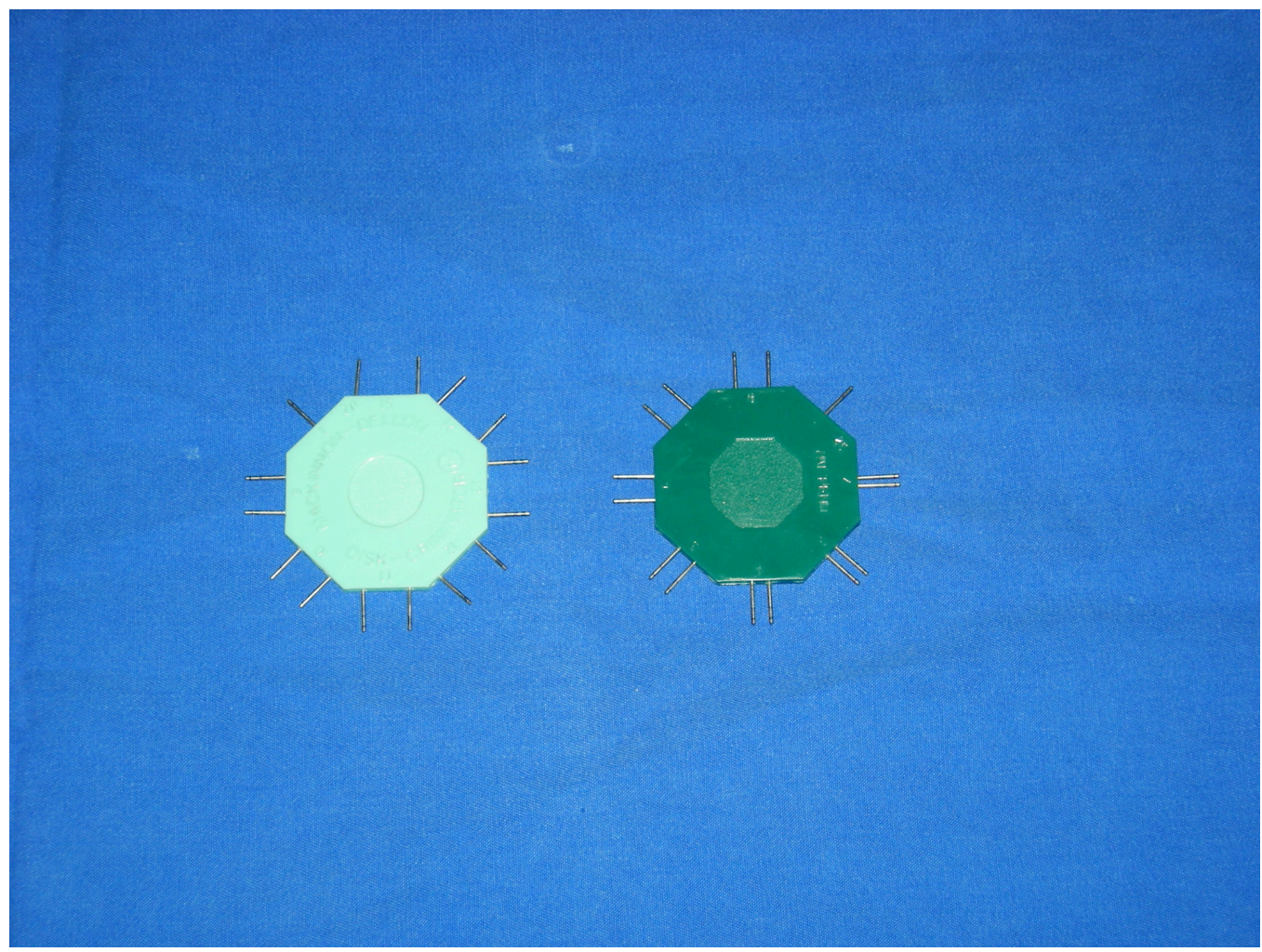

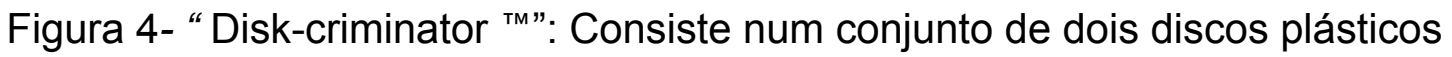
com extremidades de metal de pontas rombas com distancias prédeterminadas variando entre 2 e $20 \mathrm{~mm}$. 
A seguir procedeu-se o exame dos limiares cutâneos de pressão. $O$ aparelho deve ser calibrado para a gravidade no início de cada exame. $\mathrm{O}$ examinador exerce pressão contínua e crescente da extremidade de metal sobre a polpa digital. No momento em que o estímulo passar a ser percebido o paciente aperta um botão(ligado a um aviso sonoro) e este valor é armazenado pelo computador.

São realizadas dez tentativas, e destas, as três consideradas mais adequadas são escolhidas pelo computador. A média aritmética das três medidas escolhidas é calculada, e este valor é considerado como resultado. São registradas medidas para o teste estático de um ponto (1 PE), onde o examinador exerce pressão gradual e contínua sem movimentar o aparelho, medindo portanto a função das fibras de adaptação lenta para o tato superficial. A seguir, para medir a função das fibras de adaptação rápida, o examinador realiza movimento de pressão gradual porém com uma das extremidades do aparelho em movimento. Desta forma registra-se o teste dinâmico de um ponto(1 PD).

Seguem-se os testes de limiar cutâneo de pressão de dois pontos, a saber: o teste estático de dois pontos (2 PE) e o teste dinâmico de dois pontos (2 PD). Para estes exames, a distância entre as duas extremidades do aparelho será ajustada, em mm, conforme o resultado obtido no teste de discriminação de dois pontos previamente descrito. Da mesma maneira, as duas pontas são mantidas na mesma posição ou em movimento, para medir a função das fibras de adaptação lenta ou rápida, respectivamente. Deve-se enfatizar ao paciente 
no caso dos testes de dois pontos que ele deverá acionar o botão apenas no momento em que estiver sentindo "duas pontas separadas" na polpa digital, e não no momento em que começar a sentir o estímulo de pressão. O exame completo leva cerca de 40 minutos.

O grupo controle foi dividido em pacientes com menos de 45 anos e pacientes com mais de 45 anos, de acordo com o trabalho realizado por Dellon com dados normativos da população americana. ${ }^{36,37}$ Calcularam-se a média, desvio-padrão e o intervalo de confiança de $99 \%$.

Os resultados obtidos nos grupos com síndrome do túnel do carpo nos quatro parâmetros( $1 \mathrm{PE}, 1 \mathrm{PD}, 2 \mathrm{PE}, 2 \mathrm{PD})$ foram classificados como normais ou não, de acordo com valores determinados pelo grupo controle; e comparados entre si através do teste exato de Fisher. Foram considerados anormais valores acima daqueles encontrados no intervalo de confiança de $99 \%$ do grupo controle. Foi calculada também a sensibilidade dos testes tanto estáticos quanto dinâmicos em relação à melhora clínica dos pacientes após o tratamento cirúrgico. 
Resultados 


\section{Resultados}

Os resultados dos testes de sensibilidade cutânea nos pacientes do grupo 1(sem patologia) estão resumidos na tabela 1.

Os pacientes com menos de 45 anos apresentaram média de 0,364 $\mathrm{g} / \mathrm{mm}^{2}$ no teste estático de um ponto. No teste dinâmico de um ponto a média foi de $0,404 \mathrm{~g} / \mathrm{mm}^{2}$. Nos testes estático e dinâmico de dois pontos, as médias foram de $0,536 \mathrm{~g} / \mathrm{mm}^{2}$ e de 0,5052 respectivamente.

Os pacientes com mais de 45 anos apresentaram médias de 0,508 $\mathrm{g} / \mathrm{mm}^{2}$ e de $0,489 \mathrm{~g} / \mathrm{mm}^{2}$ nos testes estático e dinâmico de um ponto respectivamente. No teste estático de dois pontos a média foi de $0,564 \mathrm{~g} / \mathrm{mm}^{2}$, enquanto que no teste dinâmico de dois pontos a média foi de $0,588 \mathrm{~g} / \mathrm{mm}^{2}$.

\begin{tabular}{llllll}
\hline \multicolumn{5}{l}{ Tabela 1- Valores dos limiares cutâneos de pressão $\left(\mathbf{g} / \mathbf{m m}^{2}\right)$} & em pacientes do Grupo 1 \\
\hline \multirow{2}{*}{$<45$} & 1 PE & 1 PD & 2 PE & 2 PD \\
anos & Média & 0,364 & 0,404 & 0,536 & 0,5052 \\
& Desvio-padrão & 0,118 & 0,16 & 0,246 & 0,173 \\
& Intervalo de confiança & 0,442 & 0,509 & 0,698 & 0,619 \\
& $99 \%$ & & & \\
$>45$ & Média & 0,508 & 0,489 & 0,564 & 0,588 \\
anos & Desvio-padrão & 0,257 & 0,241 & 0,236 & 0,254 \\
& Intervalo de confiança & 0,688 & 0,658 & 0,73 & 0,767 \\
& $99 \%$ & & & \\
\hline
\end{tabular}

NOTA: 1PE- teste estático de um ponto, 1PD- teste dinâmico de um ponto, 2PE- teste estático de dois pontos. 2PD- teste dinâmico de dois pontos 
Os resultados dos testes nos pacientes dos grupos 2 e 3 estão na tabela

2.

A sensibilidade dos mesmos grupos para o diagnóstico de síndrome do túnel do carpo está na tabela 3 .

O teste estático de um ponto foi anormal em $95,4 \%$ dos pacientes do grupo 2, e em apenas $54,5 \%$ dos pacientes do grupo 3.

O teste dinâmico de um ponto foi anormal em $100 \%$ dos pacientes do grupo 2 e em $47,6 \%$ dos pacientes do grupo 3.

O teste estático de dois pontos foi anormal em 100\% dos pacientes dialíticos, e em 95,2\% dos pacientes não portadores de doença renal crônica.

\begin{tabular}{|c|c|c|c|c|c|}
\hline \multicolumn{6}{|c|}{ Tabela 2- Valores dos limiares cutâneos de pressão $\left(\mathrm{g} / \mathrm{mm}^{2}\right)$ nos grupos 2 e 3} \\
\hline & \multicolumn{2}{|c|}{ Testes estáticos } & \multicolumn{2}{|c|}{ Testes dinâmicos } & \multirow{2}{*}{$\begin{array}{l}\text { Discriminação de } \\
\text { dois pontos }\end{array}$} \\
\hline & $1 \mathrm{PE}$ & $2 \mathrm{PE}$ & $1 \mathrm{PD}$ & $2 \mathrm{PD}$ & \\
\hline $\begin{array}{l}\text { Média total } \\
(\mathrm{g} / \mathrm{mm} 2)\end{array}$ & 2,255 & 3,532 & 1,59 & 3,079 & $6,14 \mathrm{~mm}$ \\
\hline $\begin{array}{l}\text { Média grupo } \\
2(\mathrm{~g} / \mathrm{mm} 2)\end{array}$ & 3,36 & 3,455 & 2,11 & 3,453 & $6,61 \mathrm{~mm}$ \\
\hline $\begin{array}{l}\text { Média grupo } \\
3(\mathrm{~g} / \mathrm{mm} 2)\end{array}$ & 1,875 & 3,605 & 1,04 & 2,705 & $5,6 \mathrm{~mm}$ \\
\hline
\end{tabular}


Tabela 3- Sensibilidade para o diagnóstico de síndrome do túnel do carpo utilizando o PSSD para os grupos 2 e 3

\begin{tabular}{lllll}
\hline & \multicolumn{2}{l}{ Testes estáticos } & \multicolumn{2}{l}{ Testes dinâmicos } \\
& 1PE & $2 \mathrm{PE}$ & 1 PD & 2PD \\
& & & & \\
Sensibilidade total & $76,7 \%$ & $97,6 \%$ & $74,4 \%$ & $95,2 \%$ \\
Sensibilidade & $95,4 \%$ & $100 \%$ & $100 \%$ & $100 \%$ \\
grupo 2 & & & $90,4 \%$ \\
Sensibilidade & $54,5 \%$ & $95,2 \%$ & $47,6 \%$ & \\
grupo 3 & & & & \\
\hline
\end{tabular}

Por fim, o teste dinâmico de dois pontos foi anormal em 100\% dos pacientes do grupo 2 e em 90,4\% dos pacientes do grupo 3 .

Nos testes de um ponto estático e um ponto dinâmico os pacientes do grupo 2 apresentaram uma maior sensibilidade para a detecção da síndrome do túnel do carpo em relação aos pacientes do grupo 3; com diferença estatisticamente significativa( Tabela 4).

Tabela 4- Valores de p para o teste exato de Fisher, utilizado para comparação entre os grupos 2 e 3. Houve diferença estatisticamente significativa para os testes 1 PE e 1PD

\begin{tabular}{ll}
\hline Teste & Grupo $2 \times$ Grupo 3 \\
$1 \mathrm{PE}$ & $\mathrm{p}=0,0036$ \\
$1 \mathrm{PD}$ & $\mathrm{p}=0,0001$ \\
$2 \mathrm{PE}$ & $\mathrm{p}=0,4884$ \\
$2 \mathrm{PD}$ & $\mathrm{p}=0,2326$
\end{tabular}




\section{Discussão}




\section{Discussão}

Os pacientes com insuficiência renal crônica dependentes de hemodiálise podem desenvolver síndrome do túnel do carpo, por mecanismos ainda não bem conhecidos. A verdadeira patogênese não é totalmente compreendida, e possivelmente multifatorial. Entre as hipóteses, a fístula arteriovenosa é citada por levar à isquemia segmentar do nervo mediano devido a distúrbios hemodinâmicos. Outras hipóteses levantadas são a existência de neurite causada por compressão externa do nervo mediano ou o edema induzido pela própria anastomose. Estudos demonstraram a associação entre o depósito da $\beta 2$ - microglobulina no túnel do carpo e o aparecimento da condição. A incidência da síndrome aumenta segundo o número de anos em que o paciente é submetido à hemodiálise. ${ }^{15,16,43,44}$

O diagnóstico de síndrome do túnel do carpo é considerado pela maioria dos especialistas como clínico. ${ }^{1,18,20,45,46,47}$ No entanto, nem sempre os sinais e sintomas são claramente indicativos da condição; e nestas situações deve-se lançar mão de exames complementares. Os testes eletrodiagnósticos são os mais utilizados, e considerados o padrão- ouro por alguns autores. ${ }^{21,48}$ Porém um teste eletrodiagnóstico negativo não deve ser considerado como absoluto para exclusão do diagnóstico de síndrome do túnel do carpo, assim como a positividade de um teste sem a presença de sintomas não deve levar à 
indicação de cirurgia. Isto porque a incidência de falsos negativos é de até $15 \%$ em estudos clínicos. ${ }^{1,18,46}$

Buch-Jaeger e Foucher, em estudo prospectivo envolvendo 112 pacientes com sintomas compatíveis com síndrome do túnel do carpo encontraram testes de condução nervosa positivos em apenas $61 \%$ dos pacientes. O estudo foi também incapaz de correlacionar diversos sinais clínicos avaliados como parestesia no território do nervo mediano, dor ocasional, parestesia noturna, teste de Phalen, teste de Tinel, teste de Durkan, teste de Gilliat, sensibilidade vibratória, discriminação de dois pontos e monofilamentos de Semmes-Weinstein; com os estudos eletrodiagnósticos. ${ }^{48}$

De Smet, em meta-análise realizada chega a conclusões semelhantes: não há critérios diagnósticos precisos. A eletroneuromiografia é necessária apenas em situações duvidosas, sem história e exame físico típicos. No entanto, esta não apresenta boa correlação com a gravidade dos sintomas, e nem tampouco está relacionada com prognóstico dos pacientes. ${ }^{45}$

Schrijver et al. em estudo multicêntrico randomizado, concluíram não haver indicação de realização de estudos de condução nervosa na prática clínica para avaliar os efeitos do tratamento da síndrome do túnel do carpo; uma vez que existe correlação apenas modesta entre os achados eletrodiagnósticos e a clínica. ${ }^{46}$

Graham et al. questionam a escolha dos estudos eletrodiagnósticos como padrão-ouro para o diagnóstico da síndrome do túnel do carpo, e enumera três razões para tal. Primeiro, os estudos eletrodiagnósticos tornam-se 
anormais apenas quando a compressão já é suficientemente severa para causar anormalidades estruturais no nervo mediano; em segundo lugar porque os testes de condução nervosa podem ser normais a despeito da presença de sinais e sintomas de compressão. E além disso os valores considerados anormais para medidas da condução nervosa são altamente variáveis na literatura médica. ${ }^{23,47}$

O critério adotado para a confirmação da existência da síndrome do túnel do carpo foi a melhora dos sintomas após a cirurgia, mesmo critério adotado por Szabo et al . Este critério coloca uma variável de confusão ao estudo, já que pode haver o efeito placebo na melhora dos sintomas. ${ }^{20}$ Entendemos no entanto, que este critério seja melhor do que adotar como padrão-ouro o estudo de condução nervosa, com limitações já colocadas anteriormente. Outra fraqueza de nosso estudo foi a ausência de pacientes que poderiam ser incluídos como falsopositivos. Isto deve-se possivelmente ao fato de nosso serviço ser de atenção terciária, onde normalmente os pacientes são encaminhados na maior parte das vezes com o diagnóstico realizado e com teste de condução nervosa.

Gousheh et al., em estudo com 279 pacientes, demonstraram haver uma incidência ainda maior de falsos negativos nos estudos eletrodiagnósticos em pacientes hemodialíticos com síndrome do túnel do carpo, questionando a validade dos resultados destes neste tipo de paciente. ${ }^{17}$

A literatura sobre a utilização de testes de sensibilidade cutânea no diagnóstico da síndrome do túnel do carpo é escassa e contraditória. A maior 
parte dos estudos utiliza o teste de monofilamentos de Semmes- Weinstein. Este por sua vez, apresenta problemas como a impossibilidade de realizar medidas contínuas, o fato do conjunto de monofilamentos aplicados à pele ter diversos diâmetros( e portanto, teoricamente podendo estimular um número diferente de fibras dependendo do filamento utilizado) e por fim a necessidade de cálculos matemáticos para determinação da pressão. Todos estes fatores podem levar a erros tanto de medida quanto de interpretação segundo alguns autores. ${ }^{31,33,34}$

Szabo et al. avaliaram a sensibilidade das mãos em 23 pacientes com síndrome do túnel do carpo através de vibrometria, monofilamentos de Semmes-Weinstein e teste de discriminação de dois pontos. A vibrometria encontrava-se alterada em $87 \%$ dos pacientes, os monofilamentos em $83 \%$ e o teste de discriminação de dois pontos em apenas em apenas $22 \%$ dos casos. ${ }^{49}$

Koris et al. propuseram a utilização de um teste combinando a flexão do punho e a posterior medida dos valores para monofilamentos. Os autores propõem que o aumento da isquemia neural proporcionado pela flexão do punho causaria a precipitação de alterações sensitivas identificáveis através dos testes de limiar. O teste assim configurado apresenta sensibilidade de $82 \%$ e especificidade de $86 \%{ }^{50}$

A discriminação de dois pontos apresenta boa correlação com a função da mão, no entanto começa a alterar-se apenas tardiamente na lesão compressiva dos nervos periféricos. Além disso, o fato de não haver controle da 
pressão exercida na área examinada pode levar a dificuldades de comparações entre diferentes exames realizados. ${ }^{31,32,36,51}$

Dellon et al. descreveram o PSSD como forma de associar os conceitos de limiar cutâneo de pressão e de discriminação de dois pontos. Cada um destes parâmetros avaliará aspectos diferentes da função do nervo periférico, com vantagens únicas como a medida contínua do limiar cutâneo de pressão(diferentemente do teste de Semmes- Weinstein, que como dito anteriormente mede intervalos de valores) e a possibilidade de controlar a pressão exercida na pele pelo aparelho na medida da discriminação de dois pontos; desta maneira acrescentando precisão (não medimos mais apenas a distância entre dois pontos, mas sim a menor pressão em que há percepção de dois pontos e não de apenas um). Desta maneira a aferição da discriminação de dois pontos passa a ser também um método de avaliação de limiar cutâneo, uma vez que para as medidas de dois pontos, tanto estático quanto dinâmico, estamos realizando medidas $\mathrm{em} \mathrm{g} / \mathrm{mm}^{2}$.

Este novo tipo de parâmetro utilizado( os testes de dois pontos, tanto estático quanto dinâmico), provou ter boa correlação com testes de função de mão como o Mayo Dexterity Test e o teste de reconhecimento de objetos cronometrado. $^{52}$

Essas novas modalidades descritas mostraram-se também mais sensíveis para o diagnóstico das síndromes compressivas do que os tradicionais testes utilizados. ${ }^{37}$ 
Mais ainda: o fato do PSSD estabelecer valores objetivos ao diagnóstico das síndromes compressivas do nervo periférico, abre perspectivas quanto a tentar estabelecer gradações para a doença, e a partir daí determinar o tratamento mais eficaz. ${ }^{51,53}$

Da mesma maneira, nas neuropatias secundárias a Hanseníase, neuropatias compressivas no diabético e em pacientes com neuropatias compressivas pós trauma, o PSSD mostrou-se extremamente útil no diagnóstico dessas patologias. ${ }^{51,53,54,55,56}$

Os resultados deste estudo mostraram semelhança com o realizado por Dellon et al. ${ }^{37}$, com pacientes portadores de síndromes compressivas do membro superior. Os parâmetros mais eficazes para o diagnóstico da síndrome do túnel do carpo foram os testes de dois pontos, tanto estático quanto dinâmico ( 2PE e 2 PD).

No entanto, os testes de um ponto(1 PE e 1 PD) foram mais sensíveis para o diagnóstico da síndrome do túnel do carpo em pacientes do grupo 2 , renais crônicos; quando comparados com pacientes do grupo 3. Estes dados podem refletir diferenças na gravidade da síndrome nos pacientes do grupo 2 , visto que a fisiopatologia da síndrome nestes pacientes é diferente, e relacionada ao acúmulo de $\beta 2$ - microglobulina do ligamento carpal transverso ${ }^{16}$.

Desta maneira, o PSSD pode servir como alternativa aos testes eletrodiagnósticos em pacientes com síndromes compressivas nos membros superiores, em especial naqueles portadores de insuficiência renal crônica, 
onde a literatura demonstra haver um índice elevado de falsos negativos. ${ }^{17}$ Além disso, o fato do teste de sensibilidade cutânea ser menos desconfortável para o paciente pode facilitar um controle de tratamento com melhor adesão, tendo em vista uma menor necessidade de repetição de testes eletrodiagnósticos.

A possibilidade de medir independentemente subtipos diferentes de fibras associadas à sensação de tato superficial, pode permitir acompanhamento mais fidedigno do controle de tratamento das patologias dos nervos periféricos, inclusive com direcionamento mais específico para a reabilitação sensitiva caso esta seja necessária. Novos estudos devem ser realizados para avaliar este tipo de benefício.

Os testes eletrodiagnósticos são criticados por não apresentarem correlação com a clínica, tornando difícil sua utilização para classificação de gravidade e consequentemente escolha de planejamentos terapêuticos diferenciados(cirurgia ou tratamento conservador). No entanto em nosso meio, a realização de exame comprovando existência de síndrome compressiva é por muitas vezes obrigatória, tanto para fins trabalhistas quanto para autorização de tratamento pelas empresas de medicina complementar. A inexistência de padrão-ouro para o diagnóstico de síndrome do túnel do carpo, com cerca de $15 \%$ de falsos negativos com a eletroneuromiografia pode prejudicar pacientes que se encontrem nesse grupo. Desta forma, é interessante haver uma alternativa para os testes eletrodiagnósticos. 
Além disso a existência de um exame de sensibilidade com valores objetivos e contínuos abre perspectivas para a criação de uma escala de gravidade da compressão periférica, além de possibilidade de realizar novas avaliações com dados objetivos sobre a melhora clínica do paciente. Isso já foi estabelecido por Dellon et al. em seus estudos sobre a neuropatia compressiva em pacientes diabéticos e aplicado com sucesso ${ }^{53,54}$.

Outras situações podem eventualmente beneficiar-se deste tipo de exame, como por exemplo as síndromes compressivas do nervo ulnar no túnel cubital, que sabidamente apresentam alto índice de falsos negativos nos estudos eletrodiagnósticos. ${ }^{37,57}$

Ainda não estão estabelecidas as indicações precisas para os testes de sensibilidade cutânea, mas certamente faz-se urgente a universalização e padronização do tipo de dados coletados e seu estudo para as patologias dos nervos periféricos, sejam elas compressivas ou traumáticas. A busca pela otimização do tratamento passa hoje em dia não só pela recuperação motora efetiva, mas também por um restabelecimento satisfatório da sensibilidade cutânea. Para que protocolos de tratamento eficientes possam ser estabelecidos, faz-se necessário um acompanhamento mais adequado da recuperação sensitiva em todos os seu aspectos. O testes eletrodiagnósticos continuarão a ter sua importância, no entanto condução nervosa não significa necessariamente função. Dentro deste contexto, os testes de sensibilidade cutânea devem ser realizados, em busca de um direcionamento maior na reeducação sensitiva e portanto na recuperação da função global da mão. 
Conclusões 


\section{Conclusões}

No exame com um ponto estático(1 PE), os pacientes com síndrome do túnel do carpo(com diagnóstico confirmado) em hemodiálise crônica apresentaram resultados anormais em quantidade maior que os pacientes com síndrome do túnel do carpo sem doença renal, de maneira estatisticamente significativa.

No exame com um ponto dinâmico (1 PD), os pacientes com síndrome do túnel co carpo relacionada à hemodiálise também apresentaram exames anormais em quantidade maior que pacientes com a síndrome do túnel do carpo mas sem doença renal. Esta diferença também foi estatisticamente significativa.

Nos testes com dois pontos, tanto estático quanto dinâmico (2 PE e 2 PD), não houve diferença estatística entre a quantidade de exames anormais dos grupos 2 e 3 . 
Anexos 


\section{ANEXO A \\ TERMO DE CONSENTIMENTO LIVRE E ESCLARECIDO \\ I - DADOS DE IDENTIFICAÇÃO DO SUJEITO DA PESQUISA OU RESPONSÁVEL LEGAL}

1. NOME DO PACIENTE

DOCUMENTO DE IDENTIDADE N ${ }^{\circ}$ :

SEXO : .M F

DATA NASCIMENTO:

ENDEREÇO

$\mathrm{N}^{\circ}$

APTO:

BAIRRO:

CIDADE

CEP:

TELEFONE (.......)

2.RESPONSÁVEL LEGAL

NATUREZA(grau de parentesco, tutor, curador)

DOCUMENTO DE IDENTIDADE :

SEXO: $M \quad F$

DATA NASCIMENTO:

...............

ENDEREÇO:

$\mathrm{N}^{\circ}$

APTO:

BAIRRO:

CEP:

CIDADE:

TELEFONE:DDD

)

\section{II - DADOS SOBRE A PESQUISA CIENTÍFICA}

1. TÍTULO DO PROTOCOLO DE PESQUISA Avaliação da Sensibilidade Cutânea em pacientes com Síndrome do Túnel do Carpo em programa de hemodiálise

PESQUISADOR: Hugo Alberto Nakamoto

CARGO/FUNÇÃO: Médico INSCRIÇÃO CONSELHO REGIONAL № 90807

UNIDADE DO HCFMUSP: Disciplina de Cirurgia Plástica do Departamento de Cirurgia da FMUSP.

3. AVALIAÇÃO DO RISCO DA PESQUISA:

$\begin{array}{lcc}\text { SEM RISCO X } & \text { RISCO MÍNIMO } & \text { RISCO MÉDIO } \\ \text { RISCO BAIXO } & \text { RISCO MAIOR }\end{array}$

(probabilidade de que o indivíduo sofra algum dano como consequência imediata ou tardia do estudo)

4.DURAÇÃO DA PESQUISA : Dois anos 


\section{III - REGISTRO DAS EXPLICAÇÕES DO PESQUISADOR AO PACIENTE OU SEU REPRESENTANTE LEGAL SOBRE A PESQUISA CONSIGNANDO:}

1. justificativa e os objetivos da pesquisa

Os objetivos desta pesquisa são avaliar a sensibilidade da pele da mão de pacientes com Síndrome do Túnel do Carpo em programa de hemodiálise antes e após um ano do tratamento cirúrigico, e comparar os resultados com pacientes portadores da mesma doença porém sem associação com programa de hemodiálise. A síndrome do Túnel do carpo é uma doença que acomete o nervo mediano da mão, que pode ficar apertado na região do pulso devido a diversas causas, entre elas a realização de hemodiálise durante um longo período de tempo. Os sintomas relacionados a este acometimento do nervo são na maior parte das vezes formigamentos ou dormência na mão, perda da sensibilidade na ponta dos dedos e às vezes perda de força.

2. procedimentos que serão utilizados e propósitos, incluindo a identificação dos procedimentos que são experimentais

-Avaliação da Sensibildade da pele da mão antes do tratamento cirúrgico e após um ano do tratamento- $O$ paciente será submetido a um exame que testará como está a sensibilidade da sua mão, antes e depois de um ano da cirurgia. O exame realizado não é doloroso, e não utiliza agulhas. O objetivo do exame é conseguir quantificar a melhora da sensibilidade da mão depois da realização da cirurgia, para mostrar que este tipo de exame é útil para o acompanhamento da evolução e do controle do tratamento desta doença.

- Liberação cirúrgica do túnel do carpo- o paciente será submetido a uma operação, com um corte na região do pulso para a liberação do nervo mediano que está apertado neste local, causando os sintomas citados anteriormente. A cirurgia é simples, e na maior parte das vezes o paciente pode ir para casa no mesmo dia. 
3. desconfortos e riscos esperados

Os riscos esperados são os relacionados ao procedimento cirúrgico, a saber: infecção no local da cirurgia, lesão intra-operatóriado nervo mediano, lesões nos tendões da mão. Todas as complicações são raras se a cirurgia for realizada de maneira adequada.

4. benefícios que poderão ser obtidos

Será possível através deste estudo analisar o comportamento da síndrome do túnel do carpo nos pacientes submetidos à hemodiálise, que é uma população com características diferentes da maioria das pessoas que apresentam este problema. Com isso, esperamos que seja possível descobrir a doença mais cedo e estabelecer diferenças no tipo de tratamento realizado em pacientes submetidos à hemodiálise.

5. procedimentos alternativos que possam ser vantajosos para o indivíduo

$\mathrm{Na}$ fase inicial da doença- pode-se tratar a doença em sua fase inicial com a ajuda de aparelhos de imobilização da mão que propiciem uma posição em que a pressão sobre o nervo no pulso não seja tão grande, ajudando o nervo então a desinchar sozinho. 


\section{IV - ESCLARECIMENTOS DADOS PELO PESQUISADOR SOBRE GARANTIAS DO SUJEITO DA PESQUISA CONSIGNANDO:}

1. acesso, a qualquer tempo, às informações sobre procedimentos, riscos e benefícios relacionados à pesquisa, inclusive para dirimir eventuais dúvidas.

O paciente poderá, a qualquer tempo dispor de informações relativas aos procedimentos(exame de sensibilidade e tratamento cirúrgico), aos riscos do tratamento e benefícios relacionados a este estudo; inclusive para o esclarecimento de dúvidas

2. liberdade de retirar seu consentimento a qualquer momento e de deixar de participar do estudo, sem que isto traga prejuízo à continuidade da assistência.

O paciente terá direiro a deixar de participar do estudo a qualquer momento que quiser, sem prejuízo no seu tratamento médico

3. salvaguarda da confidencialidade, sigilo e privacidade.

O paciente terá garantia de que seus dados pessoais e médicos serão confidenciais, interessando somente ao mesmo e ao médico responsável pela pesquisa.

4. disponibilidade de assistência no HCFMUSP, por eventuais danos à saúde, decorrentes da pesquisa.

O paciente tem garantida a assistência médica por eventuais danos à sua saúde de forem estes decorrentes da pesquisa.

5. viabilidade de indenização por eventuais danos à saúde decorrentes da pesquisa. 


\section{INFORMAÇÕES DE NOMES, ENDEREÇOS E TELEFONES DOS RESPONSÁVEIS PELO ACOMPANHAMENTO DA PESQUISA, PARA CONTATO EM CASO DE INTERCORRÊNCIAS CLÍNICAS E REAÇÕES ADVERSAS.}

Em caso de dúvidas, favor entrar em contato com Dr. Hugo Alberto Nakamoto Celular 82593122

Alameda Casa Branca, 806 1. Andar telefone 3088-9333

\section{VI - CONSENTIMENTO PÓS-ESCLARECIDO}

Declaro que, após convenientemente esclarecido pelo pesquisador e ter entendido o que me foi explicado, consinto em participar do presente Protocolo de Pesquisa

São Paulo, de de 200 .

assinatura do sujeito da pesquisa ou responsável legal

assinatura do pesquisador

(carimbo ou nome Legível) 


\section{ANEXO B}

\section{LISTA DE PACIENTES}

\begin{tabular}{|c|c|c|c|}
\hline GRUPO 1 & INICIAIS & SEXO & IDADE \\
\hline 1 & JCS & MASC & 78 \\
\hline 2 & ALS & MASC & 71 \\
\hline 3 & EM & MASC & 77 \\
\hline 4 & CS & FEM & 65 \\
\hline 5 & PT & FEM & 50 \\
\hline 6 & AMS & FEM & 62 \\
\hline 7 & GV & MASC & 70 \\
\hline 8 & IS & MASC & 52 \\
\hline 9 & $\mathrm{JN}$ & MASC & 59 \\
\hline 10 & NG & MASC & 54 \\
\hline 11 & JPR & MASC & 52 \\
\hline 12 & TB & MASC & 22 \\
\hline 13 & RFI & MASC & 20 \\
\hline 14 & FJA & FEM & 20 \\
\hline 15 & BM & MASC & 29 \\
\hline 16 & $\overline{A L}$ & MASC & 21 \\
\hline 17 & RG & MASC & 19 \\
\hline 18 & RBC & MASC & 20 \\
\hline
\end{tabular}




\begin{tabular}{|c|c|c|c|}
\hline GRUPO 1 & INICIAIS & SEXO & IDADE \\
\hline 19 & MS & FEM & 40 \\
\hline 20 & MY & MASC & 20 \\
\hline 21 & LI & MASC & 20 \\
\hline 22 & RK & FEM & 21 \\
\hline 23 & SY & MASC & 19 \\
\hline 24 & GL & FEM & 26 \\
\hline
\end{tabular}




\begin{tabular}{|c|c|c|c|}
\hline GRUPO 2 & INICIAIS & SEXO & IDADE \\
\hline 1 & MAP & FEM & 50 \\
\hline 2 & ILR & MASC & 41 \\
\hline 3 & ERG & MASC & 54 \\
\hline 4 & MLM & FEM & 50 \\
\hline 5 & JMS & MASC & 56 \\
\hline 6 & LCM & MASC & 48 \\
\hline 7 & ESS & MASC & 54 \\
\hline 8 & EAFR & FEM & 49 \\
\hline 9 & CGP & MASC & 59 \\
\hline 10 & MLSP & FEM & 56 \\
\hline 11 & HLS & FEM & 46 \\
\hline 12 & JCS & MASC & 64 \\
\hline 13 & ERM & MASC & 54 \\
\hline 14 & RBG & FEM & 44 \\
\hline
\end{tabular}




\begin{tabular}{|c|c|c|c|}
\hline GRUPO 3 & INICIAIS & SEXO & IDADE \\
\hline 1 & JN & FEM & 55 \\
\hline 2 & MACP & FEM & 62 \\
\hline 3 & $\overline{M C L}$ & FEM & 42 \\
\hline 4 & MFAO & FEM & 28 \\
\hline 5 & CBS & FEM & 40 \\
\hline 6 & GNS & FEM & 57 \\
\hline 7 & MBG & FEM & 77 \\
\hline 8 & TVSL & FEM & 39 \\
\hline 9 & APV & MASC & 36 \\
\hline 10 & ACB & FEM & 38 \\
\hline 11 & ZGB & FEM & 45 \\
\hline 12 & DFV & FEM & 46 \\
\hline 13 & MGSR & FEM & 55 \\
\hline 14 & ROSA & FEM & 37 \\
\hline 15 & MLA & FEM & 50 \\
\hline
\end{tabular}




\section{ANEXO C}

FICHA DE PROTOCOLO

Nome:

$R G$ : Idade: Telefones:

HMA:

Parestesia Noturna:( ) Sim ( ) Não

Perda de força; ( ) Sim ( ) Não

Evolução em anos:

Renal Crônica? ( )SIM ( ) NÃO

Anos:

Fístula: ( ) Direita ( ) Esquerda

Uso de

Medicações:

Cirurgias

Prévias:

Outras

patologias:

Exame Físico:

Tinel:

Phalen:

ENMG: 
PSSD

\begin{tabular}{|l|l|l|l|l|l|}
\hline & DISK & 1PE & 1PD & 2PE & 2PD \\
\hline Mão Direita & & & & & \\
\hline Mão Esquerda & & & & & \\
\hline
\end{tabular}




\section{Referências}




\section{Referências}

1. Akelman, E. Carpal Tunnel Syndrome. In: Berger, R; Weiss, AP. Hand Surgery. $1^{\text {st }}$ ed. Philadelphia: Lippincot Williams \& Wilkins, 2004. p. $867-$ 885

2. Paget,J. Historical Article: The first description of carpal tunnel syndrome. J Hand Surg(Br). 2007; 32:195-197.

3. Mackinnon, SE; Dellon, AL. Carpal Tunnel Syndrome. In: Surgery of the Peripheral Nerve. $1^{\text {st }}$ ed. New York: Thieme Medical Publishers, 1988. p. 148-170.

4. Phalen, GS; Gardner, WJ; LaLonde, AA. Neuropathy of the median nerve due to compression beneath the transverse carpal ligament. J Bone Joint Surg(Am). 1950; 32(1): 109-112.

5. Zachary, RB. Thenar palsy due to compression of the median nerve in the carpal tunnel. Surg Gynecol Obstet. 1945; 81: 213.

6. Rotman, MB; Manske, PR . Anatomic relationships of an endoscopic carpal tunnel device to surrounding structures. J Hand Surg (Am).1993(18): 442-450.

7. Backhouse, KM; Churchill-Davidson, D. Anomalous palmaris longus muscle producing carpal tunnel-like conpression. Hand. 1975; 7(1): 2224. 
8. Ametewee, K; Harris, A; Samuel, M. Acute carpal tunnel syndrome produced by anomalous flexor digitorum superficilais indicis muscle. J Hand Surg(Br). 1985; 10: 83-84.

9. Jabaley, M. Personal observations on the role of lumbrical muscles in carpal tunnel syndrome. J Hand Surg(Am). 1978; 3(1):82-84.

10. Baumann, TD; Gelberman, RH; Mubarak, SJ; Garfin, SR. The acute carpal tunnel syndrome. Clin Orthop Relat Res. 1981; 156: 151-156.

11. Rydevik, B; Lundborg, G. Permeability of intraneural microvessels in perineurum following acute graded experimental nerve compression. Scan J Plast Reconstr Surg. 1977; 11(3): 179-181.

12. Mackinnon, SE; Dellon, AL; Hudson, AR; Hunter, D. Chronic nerve compression: an experimental model in the rat. Ann Plast Surg. 1984; 13(2): 112-120.

13. Mackinnon, SE; Dellon, AL; Hudson, AR; Hunter, D. A primate model for chronic nerve compression. J Reconstr Microsurg. 1985; 1(3): 185-194.

14. Warren, DJ; Otieno, LS. Carpal Tunnel syndrome in patients on intermittent hemodialysis. Post grad Med 1975; 51: 450-456.

15. Buchs, JB; Zbrodowski, A. Circulatory repercussions of arteriovenous fistulae at the carpal tunnel level. J Urol. 1983; 89(3): 183-186.

16. Morita, T; Suzuki, M; Kamimura, A; Hirasawa; Y. Amyloidosis of a possible new type in patients receiving long-term hemodyalisis. Arch Path Lab Med. 1985; 109: 1029-1032. 
17. Gousheh, J; Iranpour, A. Association between Carpal Tunnel Syndrome and Arteriovenous Fistula in Hemodialysis Patients. Plast Reconstr Surg. 2004; 116(2): 508-513.

18. Hentz, VR; Lalonde, DH. CME Article: Self Assesment and Performance in Practice: The Carpal Tunnel. Plast Reconstr Surg, 2008. 12(4 Suppl): $1-10$.

19. JaJoie, AS; McCabe, SJ; Thomas, B, Edgell, SE. Determining the Sensitivity and Specificity of Commom Diagnostic Tests for Carpal Tunnel Syndrome Using Latent Class Analysis. Plast Reconstr Surg. 2005; 116(2): 502-507.

20. Szabo, RM; Slater, RR; Farver, TB; Stanton, DB; Sharman, WK. The Value of Diagnostic Testing in Carpal Tunnel Syndrome. J Hand $\operatorname{Surg}(A m) .1999 ; 24:$ 704-714.

21. Johnson, EW. Diagnosis of carpal tunnel syndrome. The gold standart [editorial]. Am J Phys Med Rehabil. 1993; 72: 1.

22. Jablecki, CK; Andary, MT; Floeter, MK. American Association of Eletrodiagnostic Medicine, American Academy of Physical Medicine and Rehabilitation. Practice parameter for eletrodiagnostic studies in carpal tunnel syndrome: summary statement. Muscle Nerve. 2002; 25: 918-922.

23. Graham. B. The Value added by eletrodiagnostic testing in the diagnosis of carpal tunnel syndrome. J Bone Joint Surg(Am). 2008; 90: 2587-2593. 
24. Gasser, HS; Erlanger, J. The Compond nature of tha action current as disclosed by the cathode ray oscilloscope. Am J Physiol. 1924; 70: 624666.

25. Mountcastle, V; Talbot, WH; Darian-Smith, I; Kornhuber, HH. Neural Basis of The sense of flutter-vibration. Science. 1967; 155: 597-600.

26. Novak, CB; Mackinnon, SE; Williams, JI, Kelly, L. Establishment of Reability in the Evaluation of Hand Sensibility. Plast Reconstr Surg. 1992; 92(2):311-322.

27. Dellon, AL. Somatosensory Testing and Rehabilitation. $1^{\text {st }}$ ed. American Occupational Therapy Association, $1^{\text {st }}$ ed. Bethesda: The American Occupational Therapy Association: 1997. Chapter 12, p. 299-343:

Specific applications \& normative data- upper extremity.

28. Moberg, E. Aspects of sensation in reconstructive surgery of the upper extremity. J Bone Joint Surg (Am). 1964; 46: 817-825.

29. Dellon, AL. The moving two point discrimination test: clinical evaluation of the quickly-adapting fiber receptor. J Hand Surg(Am). 1978: 3(5):474-481.

30. Novak, CB; Mackinnon, SE; Kelly, L. Correlation of two point discrimination and hand function following median nerve injury. Ann Plast Surg. 1993; 31(6): 495-498.

31. Bell-Krotowski, JA; Buford, WL. The force/time relationship of clinically used sensory instruments. J Hand Ther. 1997; 10(4): 297-309. 
32. Jerosch-Herold, C. Should sensory function after median nerve injury and repair be quantified using two-point discrimination as the critical measure?Scand J Plast Reconstr Hand Surg. 2000; 34(4): 339-343.

33. Dellon, AL; Mackinnon, SE; Brandt, KE. The markings of the SemmesWeinstein nylon monofilaments. J Hand Surg (Am).1993; 18: 756-757.

34. Levin, S; Pearsall, G; Ruderman, RJ. Von Frey's method of measuring pressure sensibility in tha hand: an engineering analysis of the SemmesWeinstein pressure aesthesiometer. J Hand Surg(Am).1978; 3(3): 211216.

35. Pagel, KJ; Kaul, MP; Dryden, JD. Lack of utility of Semmes-Weinstein monofilament testing in suspected carpal tunnel syndrome. Am J Phys Med Rehabil. 2002; 81: 597-600.

36. Dellon, ES; Mourey, R; Dellon, AL. Human Pressure Perception Values for Constant and Moving One- and Two- Point Discrimination. Plast Reconstr Surg 1992. 90(1): 112-117.

37. Dellon, Al; Keller, KM. Computer- Assisted Quantitative Sensorimotor Testing in Patients with Carpal and Cubital Tunnel Syndromes. Ann Plast Surg 1997. 38(5): 493-502.

38. Ferreira, MC; Costa, MP; Cunha, MS; Sakae, E; Fels, KW. Sensibility of breast after reduction mammaplasty. Ann Plast Surg. 2003; 51(1): 6-9.

39. Fogaça, WC; Ferreira, MC; Dellon, AL. Infraorbital nerve injury associated with zygoma fractures: documentation with neurosensory testing. Plast Reconstr Surg. 2004; 113(3): 834-838. 
40. Fels, KW; Cunha, MS; Sturtz, GP, Gemperli, R; Ferreira, MC. Evaluation of cutaneous abdominal wall sensibility after abdominoplasty. Aesthetic Plast Surg. 2005; 29(2): 78-82.

41. Ferreira, MC; Rodrigues, L; Fels, K. New method of cutaneous sensibility in diabetic feet: preliminary report. Rev Hosp Clin Fac Med Sao Paulo. 2004; 59(5): 286-290.

42. Mackinnon, SE; Dellon, AL. Two point discrimination tester. J Hand Surg(Am). 1985; 6(1): 906-907.

43. Naito, M; Ogata, K; Goya, T. Carpal Tunnel Syndrome in Chronic Renal Dialysis Patients: Clinical Evaluation of 62 Hands and Results of Operative Treatment. J Hand Surg (Br). 1986; 12(3): 366-374.

44. Shin, J; Nishioka, M; Shinko, S; Shibuya, K; Sugiki, M; Kasumoto, H; Fudo, A; Bito, Y; Fujita, Y; Komaba, K. Carpal Tunnel Syndrome and Plasma $\beta 2-$ microglobulin concentration in hemodialysis patients. Ther Apher Dial. 2008; 12(1): 62-66.

45. De Smet, L. Value of some clinical provocative tests in carpal tunnel syndrome: do we need electrophysiology and can we predict the outcome? Hand Clin. 2003; 19: 387-391.

46. Scrijver, HM; Gerritden, AA; Strijers, RLM; Uitdehaag, BMJ; Scholten, RJPM; de Vet, HCW; Bouter, LM. Correlating nerve conduction studies and clinical outcome measures on carpal tunnel syndrome: lessons from a randomized controlled trial. J Clin Neurophysiol. 2005; 22(3): 216-221. 
47. Graham. B; Regehr, G; Naglie, G; Wright, JG. Development and validation of diagnostic criteria for carpal tunnel syndrome. J Hand Surg (Am). 2006; 31(6): 919- 924.

48. Buch-Jaeger, N; Foucher, G. Correlation of clinical signs with nerve conduction tests in the diagnosis of carpal tunnel syndrome. J Hand Surg (Br). 1994; 19(6): 720-724.

49. Szabo, RM; Gelberman, RH; Dimick, MP. Sensibility Testing in patients with carpal tunnel syndrome. J Bone Joint Surg(Am). 1984; 66(1): 60-64.

50. Koris, M; Gelberman, RH; Duncan, K; Boublick, M; Smith, B. Carpal Tunnel Syndrome- Evaluation of a quantitative provocational test. Clin Orthop Relat Res. 1990; 251:157-161.

51. Siemionow, M; Zielinski, M; Sari, A. Comparison of Clinical evaluation and neurosensory testing in the early diagnosis of superimposed entrapment neuropathy in diabetic patients. Ann Plast Surg. 2006; 57(1): $41-49$

52. Dellon, E, Keller, KM; Moratz, V, Dellon, AL. Validation of Cutaneous pressure Threshold Measurements for the Evaluation of Hand Function. Ann Plast Surg 1997. 38(5): 485-492.

53. Caffee, $\mathrm{HH}$. Treatment of diabetic neuropathy by descompression of the posterior tibial nerve. Plast Reconstr Surg. 2000; 106(4): 813-822.

54. Dellon, AL. Treatment of Symptomatic Diabetic Neuropathy by Surgical Decompression of Multiple Peripheral Nerves. Plast Reconstr Surg. 1992; 89(4): 689-697. 
55. Seiler, DK; Wilton, J; Dellon, AL. Detection of neuropathy due to Mycobacterium leprae using noninvasive neurosensory testing of susceptible peripheral nerves. Ann Plast Surg. 2005; 55(6): 633-637.

56. Coert, JK; Meek, MF; Gibeaeult, D; Dellon, AL. Documentation of Posttraumatic Nerve Compression in Patients with Normal Electrodiagnostic Studies. J Trauma. 2004; 56(2): 338-344.

57. Husain, SN; Kaufmann, RA. The Diagnosis and Treatment of Cubital Tunnel Syndrome. Curr Opin Orthop. 2008, 19(5): 470-473. 